\title{
Multipath Rate based Congestion Control for Mobile Ad Hoc Networks
}

\author{
${ }^{1}$ Soundararajan, S. and ${ }^{2}$ R.S. Bhuvaneswaran \\ ${ }^{1}$ Department of Computer Science and Engineering, \\ Velammal Institute of Technology, Chennai, India \\ ${ }^{2}$ Ramanujan Computing Center, College of Engineering, \\ Guindy, Anna University, Chennai, India
}

\begin{abstract}
In Mobile Ad hoc networks (MANET) traditional congestion control mechanism encounters new challenges such as packet losses, bandwidth degradation and frequent link failures. Congestion degrades the performance of mobile ad hoc network (MANET) and hence it can be greatly reduced by using multipath routing and rate control techniques. In this paper, a multipath rate based congestion control algorithm is proposed. The proposed algorithm has rate estimation and rate control mechanisms in which the traffic rate is adjusted based on the estimated rate. The estimate rate can be obtained from the intermediate nodes by the destination node which in turn forward this information to the source. Simulation results show that the proposed rate control algorithm outperforms the traditional congestion control techniques in terms of throughput and packet delivery ratio.
\end{abstract}

\section{Keywords}

MANET, Congestion, Multipath Routing, Load Balancing, Rate Control

\section{INTRODUCTION}

\subsection{Mobile Ad hoc Network (MANET)}

The network comprising mobile nodes with no infrastructure is termed as mobile ad hoc networks (MANET). The selforganization of the mobile nodes results in a network over radio links. For establishing routes among nodes, some specific routing protocols are used. [1] High mobility nature of the ad hoc networks resulted in new challenge that initiates a concept of dissimilarity between wireless characteristics and nature of the network topology. The exclusive nature of the mobile ad hoc network makes it to be deployed in any place and at any occasion. [3]

The application of the mobile ad hoc network includes military battlefield circumstance, disaster relief, and rescue during emergency, discovery etc. The group related application of mobile ad hoc networks include teleconferencing, data dissemination services etc. [2]

\subsection{Congestion Control in MANET}

It is essential to adjust the data rate used by each sender in order not to overload the network, where multiple senders compete for link bandwidth. Packets are dropped when they arrive at the router and cannot be forwarded. Many packets are dropped while excessive amount of packets arrive at a network bottleneck. The packets dropped would've traveled long way and in addition the lost packets often trigger retransmissions. This intimates that even more packets are sent into the network. And so, network throughput is still more worsened by the network congestion. There are chances of congestion collapse where almost no data is delivered successfully if no appropriate congestion control is performed. [4]

Shared broadcast medium is used in mobile ad hoc networks. Medium capacity which is very inadequate is shared within all the nodes in a collision domain. While delivering data to multiple destinations, multicast communication is of great concern in these networks, since it helps saving resources. Group communication which is an inherent feature of many proposed applications in MANETs is added to this broadcast medium. So, it is important to avoid congestion collapse in wireless multihop networks in order to perform efficient congestion control. [5]

The protocol of rate control is proposed for distinctive flows of characteristics user which takes in two components such as endhost congestion control layer among IP and TCP/UDP and every router upholds a single fair share for each link. [6] The issues such as exploding nature of the network and self-locking scenario while ACK packets arrive can be avoided by rate based transmission technique. [7]

In order to meet the bandwidth and delay requirements of real time traffic, rate control is done in a localized manner at each mobile node in entirely scattered and decentralized way. The rate control is designed for restricting the best effort traffic for creating the necessary bandwidth. Rate control also permits the best effort traffic to make use of the bandwidth in efficient way that is not used by the real time traffic during any particular situation. The total rate of all best effort traffic and real-time traffic distributed over each load shared media channel is sustained below a certain threshold, for minimizing the excessive delay. [8]

The method of identifying the approved data rate is facilitated by the explicit technique of rate control mechanism and thus the flows responds quickly to modulation in bandwidth and rerouting events. [9] The requisite transmission rate for the available resource facility can be adjusted with the help of controlling the flow which avoids congestion. [10]

\subsection{Proposed Solution}

An important challenge in congestion control technique in MANET is that how the sender learns about network congestion and adjusts its rate.

This paper extends our previous work [15], which proposed congestion controlled adaptive multi-path routing protocol to achieve load balancing and avoid congestion in MANETs.

\section{RELATED WORK}

S.Karunakaran et al [1] proposed a cluster based congestion control (CBCC) protocol that consists of scalable and distributed cluster-based mechanisms for supporting congestion control in ad hoc networks. The clusters autonomously and proactively monitor congestion within its localized scope. The present approach improves the responsiveness of the system when 
compared to end-to-end techniques. After estimating the traffic rate along a path, the sending rate of the source nodes is adjusted accordingly. Thus this protocol look forward the injection of dynamic flows in the network and proactively adjusts the rate while waiting for congestion feedback.

S.Venkatasubramanian et al [8] proposed QoS architecture for Bandwidth Management and Rate Control in MANET. The proposed QoS architecture contains an adaptive bandwidth management technique which measures the available bandwidth at each node in real-time and it is then propagated on demand by the QoS routing protocol. The source nodes perform call admission control for different priority of flows based on the bandwidth information provided by the QoS routing. A rate control mechanism is used to regulate best-effort traffic, whenever network congestion is detected.

Kai Chen et al [9] proposed an explicit rate-based flow control scheme (called EXACT) for the MANET network. In EXACT, flow's allowed rate is explicitly conveyed from intermediate routers to the end-hosts in each data packet's special control header. As a result, EXACT reacts quickly and precisely to re-routing and bandwidth variation, which makes it especially suitable for a dynamic MANET network.

Kazi Chandrima Rahman et al [11] proposed explicit ratebased congestion control (XRCC) for multimedia streaming over mobile ad hoc networks. XRCC addresses the problems that TCP faces when deployed over ad-hoc networks, and thus shows considerable performance improvement over TCP. Although XRCC minimizes packet drops caused by network congestion as compared to TCP congestion control mechanism, it still suffers from packet drops.

Hongqiang Zhai et al [12] proposed a novel rate based endto-end Congestion Control scheme (RBCC). Based on the novel use of channel busyness ratio, which is an accurate sign of the network utilization and congestion status, a new rate control scheme has been proposed to efficiently and reliably support the transport service in MANET. In RBCC, a sub-layer consisting of a leaky bucket is added under TCP to control the sending rate based on the network layer feedback at the bottleneck node.

Emmanuel Lochin et al [13] proposed a complete reliable rate-based protocol based on TCP-Friendly Rate Control (TFRC) and selective acknowledgement (SACK) mechanisms. This design also introduces a flow control variable, which regulates the sender to avoid packet loss at the receiver due to a slow receiver. In this mechanism, there is no packet loss due to flow control, at the receiver, and applies a smoothness criterion to demonstrate that the introduction of the flow control inside TFRC does not alter the smoothness property of this mechanism.

Yuedong $\mathrm{Xu}$ et al [14] proposed a fully distributed congestion control algorithm to balance throughput and fairness for TCP flows in multihop ad hoc networks. The interactions between the hidden nodes and network congestion are mainly focused. A distributed algorithm to improve the end-to-end throughput, and at the same time, provide per-flow fairness by exploiting cross-layer information is proposed. In the link layer, each node uses a proportional controller to determine the ECN marking probability for the purpose of notifying incipient congestion. Then the rate based TCP sender adjusts its sending rate according to the feedbacks from the link layer.

\section{MULTIPATH RATE BASED CONGESTION CONTROL TECHNIQUE}

\subsection{Overview}

The source node forwards the data packet to the destination through the intermediate nodes. On reception of the data packet at the intermediate node, percentage of channel utilization and queue length are estimated and node is verified for congestion status. This process is repeated at every intermediate node, and finally the packet reaches the destination node. After the reception of the data packet, the destination node checks for the rate information in the packets IP header fields. Along with other essential fields, estimated rate is copied to an acknowledgement packet and sent as a feedback to the sender. The sender performs rate control according to the estimated rate obtained from the destination.

\subsection{Estimation of Metrics}

\subsubsection{Rate Estimation}

The source node forwards the data packet to the destination through the intermediate nodes. Based on the buffer status of the intermediate nodes, the packet arriving or leaving at the intermediate node is either discarded from the node or updated with the status of the node.

The rate of the incoming and outgoing packet [16] is estimated as follows.

The rate of the incoming packet $\left(\tau_{\mathrm{i}}\right)$ is estimated as the reciprocal of the arrival time interval of the incoming packet.

$$
\text { i.e. } \tau_{i}=1 / T_{i}
$$

$T_{i}$ is defined as the time interval of two consecutive packets received at the node.

The rate of the outgoing packet $\left(\tau_{\mathrm{o}}\right)$ is estimated as the reciprocal of the service time of the packet.

$$
\text { i.e. } \tau_{0}=1 / T_{0}
$$

$T_{o}$ is defined as the time interval between the time that a packet arrives at node and time that it is transmitted successfully. It is also defined as summation of the time for queue, collision, back off and transmission.

The estimation of $T_{i}$ and $T_{o}$ is performed using exponential weighted moving average (EWMA) algorithm as follows [16].

$$
\begin{aligned}
& T_{i c}=(1-\sigma) T_{i p}+\sigma\left(T_{1}-T_{p}\right) \\
& T_{O C}=(1-\varepsilon) T_{O P}+\varepsilon T_{S}
\end{aligned}
$$

where $\mathrm{T}_{\mathrm{ic}}=$ time interval of the currently arrived packet $\mathrm{T}_{\mathrm{oc}}=$ service time of current packet

$\mathrm{T}_{\mathrm{ip}}$ and $\mathrm{T}_{\mathrm{op}}$ are time interval of lastly arrived packet and service time of last packet respectively

$\mathrm{T}_{1}$ and $\mathrm{T}_{\mathrm{p}}$ are the arrival time of the last packet and penultimate packet so $T_{1}-T_{p}$ is the packet arriving interval

$\mathrm{T}_{\mathrm{s}}$ is the service time of the last outgoing packet.

$\sigma$ and $\varepsilon$ are the constants for weighing $\mathrm{T}_{\mathrm{i}}$ and To with value between 0 and 1 


\subsubsection{Estimation of Channel Utilization}

In the wireless networks, when the transmission channel is being completely utilized, it is concluded that the network congestion has occurred. The channel utilization for time interval $t$ is estimated using channel busy-time $\left(\mathrm{T}_{\mathrm{c}}\right)$ metric on a percentage scale. The channel busy-time can be computed based on the category of control frame and the rate and data frame size. [18]

Let DIFS denote the distributed inter-frame spacing

Let SIFS denote the short inter-frame spacing.

Let $\alpha(\mathrm{t})$ denote the request to send (RTS) frame.

Let $\beta(\mathrm{t})$ denote the clear to send (CTS) frame.

Let $\gamma(\mathrm{t})$ represent the acknowledgement (ACK) packet.

Let $\delta(\mathrm{t})$ represent the beacon frame.

Let $\alpha_{\text {delay }}, \beta$ delay, $\gamma_{\text {delay }}$ and $\delta_{\text {delay }}$ denotes the delay component of RTS, CTS, ACK and beacon signals respectively.

Let $D_{\text {delay }}$ and $S_{\text {delay }}$ be the delay components of DIFS and SIFS respectively.

In order to compute the busy-time of a data frame $\left(\mathrm{T}_{\mathrm{cd}}\right)$, $D_{\text {delay }}$ interval is used. For a frame of size $\mathrm{Z}$ bytes transmitted at a rate $\tau$, the channel busy time ( $\left.\mathrm{T}_{\mathrm{cd}}\right)$ is computed using Eq (5).

$$
T_{c d}=D_{\text {delay }}+d(Z)(\tau)
$$

When RTS frames arrive at the data set, the $\mathrm{T}_{\mathrm{cr}}$ for the respective frame is computed using $\mathrm{Eq}(6)$.

$$
T_{c r}=\alpha_{\text {delay }}
$$

Following the reception of RTS frame, when CTS frame is encountered in the data set, it is transmitted with $S_{\text {delay. }}$ The respective $\mathrm{T}_{\mathrm{cc}}$ is given using Eq: (7)

$$
T_{C C}=S_{\text {delay }}+\beta_{\text {delay }}
$$

Following the reception of CTS frame, when ACK frame is encountered in the data set, it is transmitted with $S_{\text {delay. }}$. The corresponding $\mathrm{T}_{\mathrm{ca}}$ is given using Eq: (8)

$$
T_{c a}=S_{\text {delay }}+\gamma_{\text {delay }}
$$

When a beacon frame is encountered in the data set, it comes first by the $D_{\text {delay }}$ interval. Its $T_{c b}$ value is computed using Eq: (9)

$$
T_{c b}=D_{\text {delay }}+\delta_{\text {delay }}
$$

Tc can be computed as the sum of the time utilized by the transmission of all data and control frames in the network and the total number of delay components at time $t$ (explained in $\mathrm{Eq}$ (10)). The delay is taken into account while estimating $T_{c}$ as the medium remains unshared among the stations in the network at time $t$.

$$
\mathrm{T}_{\mathrm{c}}(\mathrm{t})=\left(\alpha(\mathrm{t}) * \mathrm{~T}_{\mathrm{cr}}\right)+\left(\beta(\mathrm{t}) * \mathrm{~T}_{\mathrm{cc}}\right)+\left(\gamma(\mathrm{t}) * \mathrm{~T}_{\mathrm{ca}}\right)+\left(\delta(\mathrm{t}) * \mathrm{~T}_{\mathrm{cb}}\right)+(
$$

The percentage channel utilization at time t, $\mathrm{CU}(\mathrm{t})$ [18] is given using Eq (11)

$$
\% C U(t)=\frac{T_{c}(t)}{10^{6}} * 100
$$

\subsubsection{Estimation of Queue Length}

The queue length describes the total traffic load in a mobile node. In general, when excess traffic flows through the mobile node, then there will more number of packets in the interface queue. Thus average queue size $\left(\mathrm{L}_{\mathrm{Q}}\right)$ [19] is defined as the node's traffic in a long term. $\mathrm{L}_{\mathrm{Q}}$ is given using Eq (12)

$$
L_{Q}=\Psi * L_{Q o l d}+(1-\Psi) * L_{Q c}
$$

Where $\mathrm{L}_{\mathrm{Qc}}=$ current value of the queue length $\psi=$ constant in the range $[0,1]$

\subsection{Congestion Detection Algorithm}

1. The source sends the data packets to the destination through the intermediate nodes.

2. Let $\mathrm{L}_{\mathrm{Qth}}$ be the predefined threshold value of queue length.

Let $\% \mathrm{CU}_{\text {th }}$ be the predefined threshold percentage channel utilization

Upon reception of the data packets, intermediate node verifies both the queue length and channel utilization (explained in section 3.2.2 and 3.2.3) and further assigns value to $C_{b}$ as per following cases.

$$
\begin{gathered}
2.1 \text { If } \mathrm{L}_{\mathrm{Q}}>\mathrm{L}_{\mathrm{Qth}} \text { and } \% \mathrm{CU}>\% \mathrm{CU}_{\text {th }} \text {, Then } \\
\text { Set } \mathrm{C}_{\mathrm{b}}=1
\end{gathered}
$$

End if

$$
\begin{gathered}
2.2 \text { If } \mathrm{L}_{\mathrm{Q}}=\mathrm{L}_{\mathrm{Qth}} \text { and } \% \mathrm{CU}=\% \mathrm{CU}_{\text {th }} \text {, Then } \\
\text { Set } \mathrm{C}_{\mathrm{b}}=0
\end{gathered}
$$

End if

After the detection of congestion, the intermediate node estimates the new rate information using equations (13) and (14). The source updates its packet sending rate with this estimated rate. This is done after getting acknowledgement packet from receiver as feedback regarding the nodes congestion status which is described in section 3.4.

\subsection{Rate based Congestion Control Algorithm}

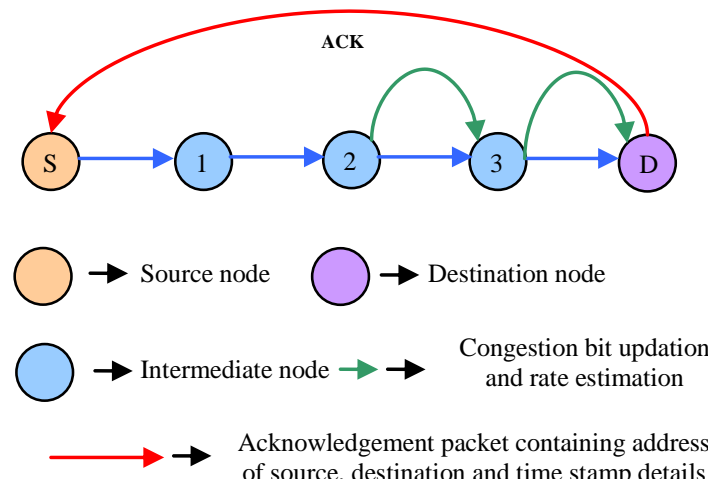

Figure-1 Congestion Control

We assume the following metrics for the rate control technique

Let $\mathrm{C}_{\mathrm{b}}=$ congestion bit set in the packets IP header

$\tau_{\mathrm{s}}=$ current rate of the sender (or) sending rate

$\tau_{\mathrm{c}}=$ current rate of the node

$\tau_{\mathrm{e}}=$ rate estimated in the intermediate node

$\tau_{\mathrm{p}}=$ estimated rate of the previous node

$\delta=$ factor which normalizes the rate value.

$\mathrm{ACK}=$ acknowledgement packet below.

The algorithm for rate based congestion control is described 
1.The current rate of the node [11] is taken as inverse of its queue length.

$$
\text { Currentrate, } \tau_{C}=1 / q
$$

Thus the rate estimated at the intermediate node $\left(\tau_{\mathrm{e}}\right)[11]$ is as follows

$$
\tau_{e}=\delta \cdot \tau_{P}+(1-\sigma) \cdot \tau_{C}
$$

where $\tau_{\mathrm{p}}$ is the estimated rate of the previous node. The factor $\sigma$ is used in order to get the smoothed value of the rate.

$$
\begin{aligned}
& 2 \text {.If } \tau_{\mathrm{e}}>\tau_{\mathrm{p}} \text { Then } \\
& \text { Set the packet with } \tau_{\mathrm{e}}
\end{aligned}
$$$$
\text { End if }
$$

3. The intermediate nodes insert the information of congestion bit $(\mathrm{Cb})$ and the estimated rate $\left(\tau_{\mathrm{e}}\right)$ into the options field of standard IP header.

The modified IP header format as defined in RFC 791 is given below:

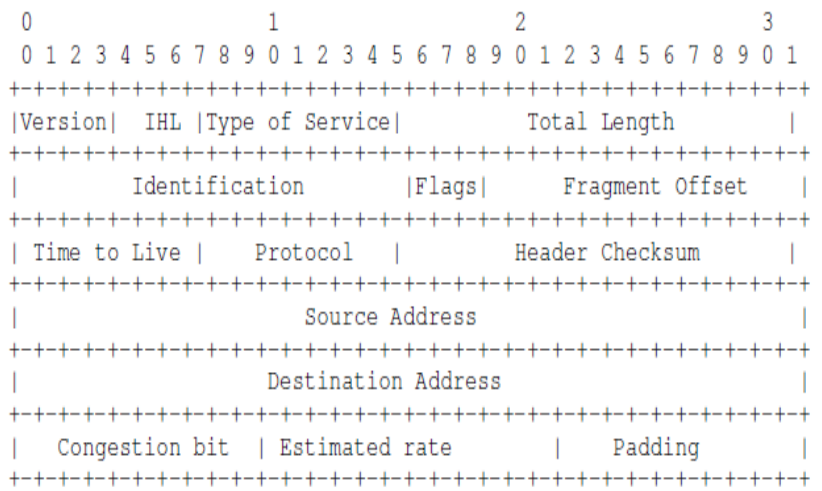

4. The process of rate estimation is repeated in every intermediate node and packets are updated with estimated rate value as per the above cases and finally the packet reaches the destination node.

5. After the reception of the data packet, the destination node checks for the value of $\mathrm{C}_{\mathrm{b}}$ and rate information in the packets IP header fields.

6. Along with $\mathrm{C}_{\mathrm{b}}, \tau_{\mathrm{e}}$ is also copied to an acknowledgement (ACK) packet and feedback to the sender by the destination node. The ACK packet contains the source address, destination address, and time stamp fields. [Shown in Figure 1]

7. After receiving an ACK packet, sender checks the value of $C_{b}$. If $C_{b}=1$, then it updates the current sending rate to the estimated rate $\tau_{\mathrm{e}}$.

$$
\text { (i.e) } \quad \tau_{\mathrm{s}}=\tau_{\mathrm{e}}
$$

Since the sending rate is adjusted based on the estimated rate from the intermediate nodes, this technique is better than the traditional congestion control technique.

\section{SIMULATION RESULTS}

\subsection{Simulation Parameters}

The proposed algorithm is experimented in the simulated environment with NS2 [17]. In this simulation, the channel capacity of mobile hosts is set to the value of 2 Mbps. We use the distributed coordination function (DCF) of IEEE 802.11 for wireless LANs as the MAC layer protocol. It has the functionality to notify the network layer about link breakage.

In our simulation, the number of nodes is fixed as 110 . The mobile nodes move in a 1250 meter x 1250 meter square region for 50 seconds simulation time. We assume each node moves independently with the same average speed. All nodes have the same transmission range of 250 meters. In our simulation, the speed is fixed as $10 \mathrm{~m} / \mathrm{s}$. For mobility, Random Way Point mobility model is used. The simulated traffic is Constant Bit Rate (CBR). We vary the traffic rate from $250 \mathrm{~kb}$ to $1000 \mathrm{~kb}$ and number of traffic flows from 2 to 8

\subsection{Performance Metrics}

The Multipath Rate Based Congestion Control (MRBCC) algorithm is compared with XRCC [11] based on the following parameters.

Average end-to-end delay: The end-to-end-delay is averaged over all surviving data packets from the sources to the destinations.

Average Packet Delivery Ratio: It is the ratio of the number of packets received successfully and the total number of packets transmitted.

Drop: It is the average number of packets dropped during the transmission.

Throughput: It is the number of packets received successfully.

The simulation results are presented in the next section

\subsection{Results}

\subsection{Based on Flow}

Initially we vary the number of flows as 2, 4, 6 and 8 keeping the rate as $250 \mathrm{~kb}$.

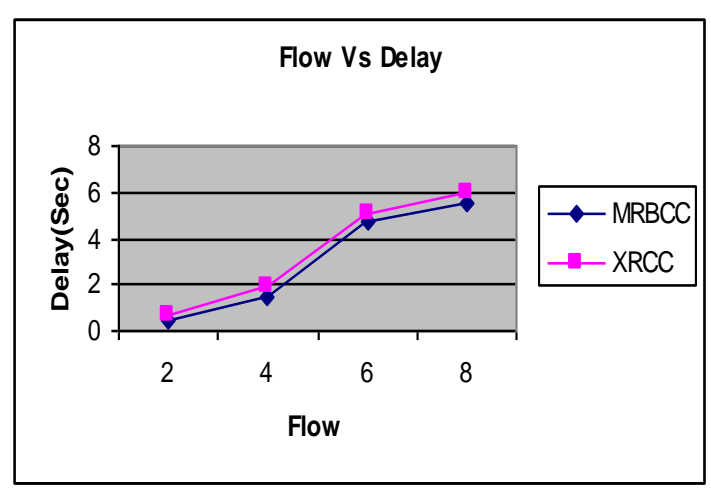

Figure 2. Flow Vs Delay

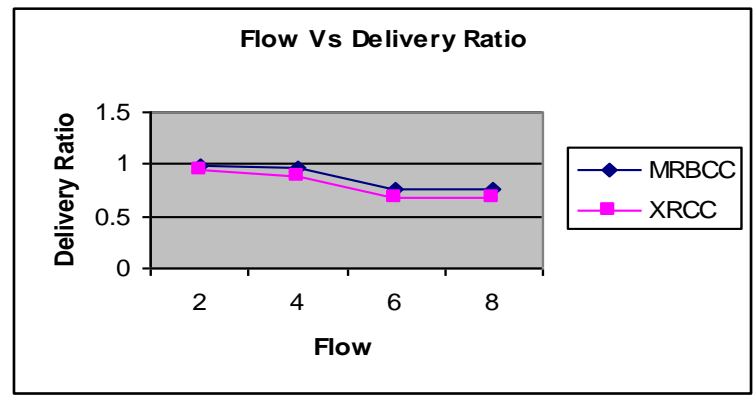

Figure 3. Flow Vs Delivery Ratio 


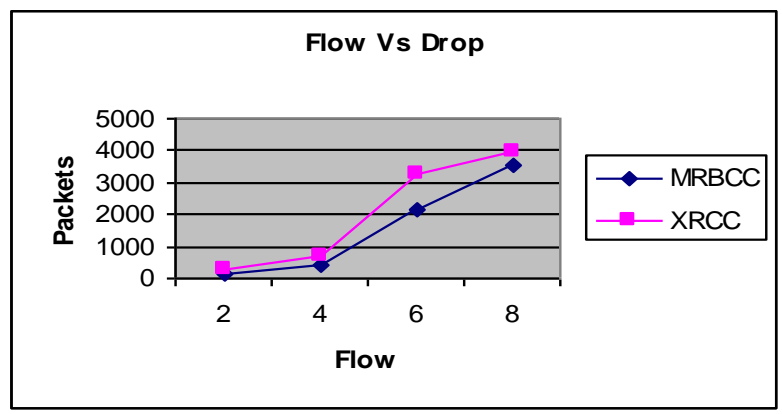

Figure 4. Flow Vs Drop

As the number of flows is increased from 2 to 8 , the incoming traffic rate will also increase leading to the overflow of queue size. So the number of packet drops increases leading to the degradation of packet delivery ratio and increased end-to-end delay. Since the rate is adjusted as per the new sending rate at each sender and also multi path routing is used, the performance of MRBCC outperforms XRCC in all the metrics.

Figure 2 shows the results of average end-to-end delay for the increasing number of flows. From the results, we can see that MRBCC scheme has $46.8 \%$ less delay than the XRCC scheme.

Figure 3 show the results of average packet delivery ratio for the varying flows scenario. Clearly our MRBCC scheme achieves $17.8 \%$ more packet delivery ratio than XRCC.

Figure 4 shows the results of packet drop versus flows. From the results, we can see that our MRBCC has $51 \%$ lesser packet drop than XRCC.

\subsubsection{Based on Rate}

In the second experiment we vary the packet sending rate value as $250,500,750$ and $1000 \mathrm{~Kb}$, keeping the no. of flows as 8 .

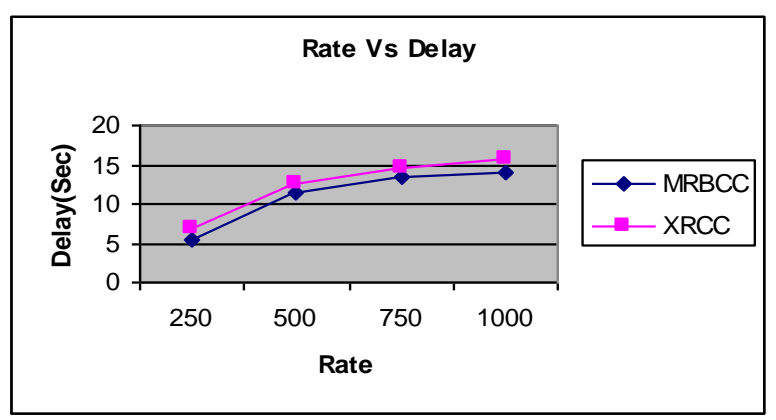

Figure 5. Rate Vs Delay

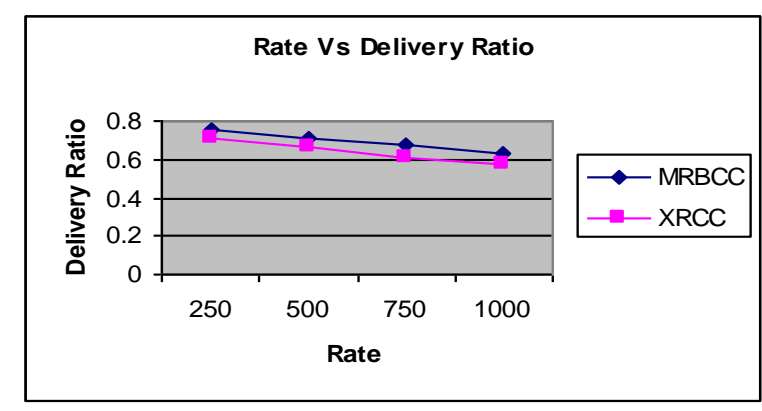

Figure 6. Rate Vs Delivery Ratio

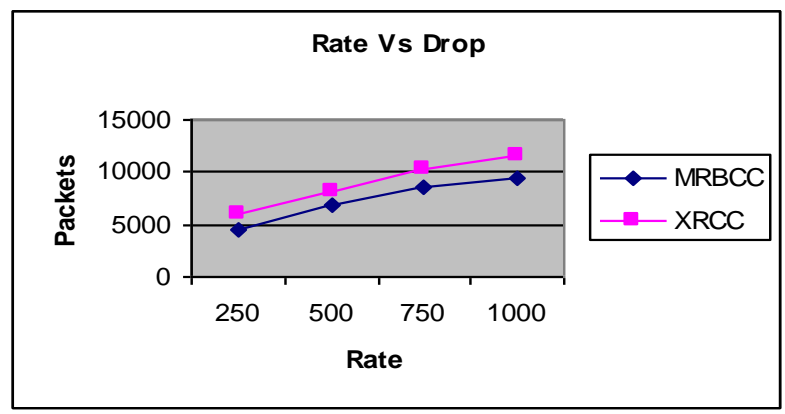

Figure 7. Rate Vs Drop

As the packet sending rate is increased from $250 \mathrm{~kb}$ to $1000 \mathrm{~kb}$, for 8 flows, the incoming traffic rate of all flows increases leading to the overflow of queue size. So the number of packet drops increases leading to the degradation of packet delivery ratio and increased end-to-end delay. In MRBCC, Since the rate is adjusted as per the new sending rate at each sender and also multi path routing is used, it reduces the packet drops significantly. So the performance of MRBCC outperforms $\mathrm{XRCC}$ in all the metrics.

Figure 5 shows the results of average end-to-end delay for the increasing the rate. From the results, we can see that MRBCC scheme has $16.11 \%$ less delay than the XRCC scheme.

Figure 6 show the results of average packet delivery ratio for the varying rate scenario. Clearly our MRBCC scheme achieves $21.4 \%$ more packet delivery ratio than XRCC.

Figure 7 shows the results of packet drop versus rate. From the results, we can see that our MRBCC has $23.01 \%$ lesser packet drop than XRCC.

\section{CONCLUSION}

In this paper, a Multipath Rate Based Congestion Control (MRBCC) algorithm is presented. In this algorithm, based on the channel utilization percentage and queue length from the intermediate nodes, congestion status and estimated rate are calculated by the destination node. The source after getting these values as a feedback from the destination, performs rate control. Simulation results using NS-2 have shown that MRBCC algorithm has higher packet delivery ratio and throughput with reduced delay, when compared with existing congestion control techniques.

\section{REFERENCES}

[1] S. Karunakaran, P. Thangaraj, "A cluster based congestion control protocol for mobile ad hoc networks", International Journal of Information Technology and Knowledge Management, Volume 2, No 2, pp. 471-474, 2010

[2] K. Tang, K. Obraczka, S. J. Lee, M. Gerla, "Congestion Controlled Adaptive Lightweight Multicast in Wireless Mobile Ad Hoc Networks", Proceedings of the Seventh IEEE Symposium on Computers and Communications (ISCC 2002), pp 967-972, July 2002.

[3] F. Alam, "Node feed-back based TCP scheme for mobile ad hoc networks", GESJ: Computer Science and Telecommunications, No 2 (31), 2011

[4] C. Lochert, B. Scheuermann, M. Mauve, "A Survey on Congestion Control for Mobile Ad-Hoc Networks", Wiley Wireless Communications and Mobile Computing 7 (5), pp. 655-676, June 2007 
[5 W. Effelsberg, M. Mauve, C. Lochert, M. Transier, B. Scheuermann, "Backpressure Multicast Congestion Control in Mobile Ad-Hoc Networks", CoNEXT 2007: Proceedings of the 3rd International Conference on Emerging Networking Experiments and Technologies, pp. 265--275, December 2007.

[6] N. Dukkipati, M. Kobayashizhang, R. Shen, N. Mckeown,, "Processor Sharing Flows in the Internet", Thirteenth International Workshop on Quality of Service (IWQoS), pp 271-285, June 2005

[7] K. Sundaresan, V. Anantharaman, H-Y. Hsieh, R. Sivakumar, "ATP: A Reliable Transport Protocol for Ad Hoc Networks", IEEE Transaction, Mobile Computing, pp588-603, 2005

[8] S.Venkatasubramanian, N.P.Gopalan, "A quality of service architecture for resource provisioning and rate control in mobile ad hoc network", International Journal of Ad hoc, Sensor \& Ubiquitous Computing (IJASUC), Vol 1, No 3, September 2010

[9] K. Chen, K. Nahrstedt, N. Vaidya, "The Utility of Explicit Rate-Based Flow Control in Mobile Ad Hoc Networks", Proc. IEEE Wireless Communications and Networking Conference (WCNC 04), pp 1921-1926, 2004.

[10] M. Belkadi, M. Lalam, A. M'zoughi, N. Tamani, M. Daoui, R.Aoudjit, "Intelligent Routing and Flow Control In MANETs", Journal of Computing and Information Technology, Volume 18, Number 3, pp 233 - 243, 2010.

[11] K. C. Rahman, S. F. Hasan, “Explicit Rate-based Congestion Control for Multimedia Streaming over Mobile Ad hoc Networks", International Journal of Electrical \& Computer Sciences IJECS-IJENS, Vol 10, No 04, 2010

[12] H. Zhai, X. Chen, Y. Fang, “ Rate-Based Transport Control for Mobile Ad Hoc Networks", IEEE Wireless
Communications and Networking Conference, pp 2264 2269, Vol 4, 2005

[13] E. Lochin, G. Jourjon, S. Ardon P. Senac, "Promoting the Use of Reliable Rate Based Transport Protocols: The Chameleon Protocol", International Journal of Internet Protocol Technology, Vol 5, Issue 4, pp 175-189, March 2010.

[14] Y. Xu, Y. Wang, C.S. Lui, D-M. Chiu, "Balancing Throughput and Fairness for TCP Flows in Multihop AdHoc Networks", 5th International Symposium on Modeling and Optimization in Mobile Ad Hoc and Wireless Networks and Workshops (WiOpt), pp 1-10, 2007

[15] S. Soundararajan, R. S. Bhuvaneswaran, "An Adaptive Multi-path Routing for Load Balancing and Congestion Control in Mobile Ad hoc Networks", Journal of computer science, volume 8, Issue 5, pp 648-655, 2012

[16] W. Wu, Z. Zhang, X. Sha, C. He, "Auto Rate MAC Protocol based on Congestion Detection for Wireless Adhoc Networks", Information Technology Journal , Volume 8, Number 8, pp 1205-1212, 2009.

[17] Network Simulator, http://www.isi.edu/nsnam/ns

[18] P. Amit, N. Jardosh Krishna, C. Ramachandran Kevin, M. Almeroth Elizabeth, " Understanding Congestion in IEEE 802.11b Wireless Networks", In Proceedings of the 2005 Internet Measurement Conference, pp 158-169,2005.

[19] X. Gao, X. Zhang, D. Shi, F. Zou, W. Zhu, " Contention and Queue-aware Routing Protocol for Mobile Ad hoc Networks", International Conference on Wireless Communications, Networking and Mobile Computing, (WiCom), pp 1628 - 1631, 2007. 